\title{
The Relationship between Serum Zinc Levels and Myopia (short-sightedness) in Korean Adolescents
}

\author{
Niamh Burke ${ }^{1}$, John S Butler ${ }^{1,2}$, Ian Flitcroft ${ }^{1,3}$ and James Loughman ${ }^{1,4}$ \\ ${ }^{1}$ Centre for Eye Research Ireland, GW205-207 Greenway Hub, Environmental Sustainability and Health Institute, \\ Technological University Dublin, Grangegorman, Dublin 7, Ireland; \\ ${ }^{2}$ School of Mathematical Sciences, College of Sciences \& Health, Dublin Institute of Technology, Kevin Street, Dublin \\ 8, Ireland; \\ ${ }^{3}$ Temple Street Children's University Hospital, Temple Street, Dublin 1, Ireland and \\ ${ }^{4}$ African Vision Research Institute, University of KwaZulu Natal, Durban, South Africa
}

Myopia, or short-sightedness is one of the leading causes of preventable blindness in the world today. Its prevalence has risen drastically over past decades, with no sign of slowing. ${ }^{(1)}$ It has been suggested that low serum zinc levels may contribute to the development of myopia, ${ }^{(2,3)}$ and therefore, the aim of this present study is to examine the relationship between serum zinc status and myopia, in adolescents, using a large nationally representative dataset.

Data from 304 adolescents aged 12-19 years, who participated in the Korean National Health and Nutrition Examination Study (KNHANES) from 2010 was used in this study. Multivariate logistic regression analysis was performed to examine the relationship between serum zinc status and myopia after adjustment for potential confounders. Additionally, the association between serum zinc and spherical equivalent refractive error (SER) was examined in the myopia group, through multiple linear regression

Among study participants, $84 \%$ were found to be myopic $(<=-0.5$ dioptres [D]). Although mean serum zinc was higher in the nonmyopes $[139.3 \mu \mathrm{g} / \mathrm{dL}]$ relative to myopes $[138.1 \mu \mathrm{g} / \mathrm{dL}]$, the difference was not statistically significant $(\mathrm{p}=0.809)$. In multiple logistic regression analyses, serum zinc was not significantly associated with myopia, after adjustment for age, gender, residence, body mass index (BMI), family income, and recreational activity. In multiple linear regression, SER was not associated with serum zinc in the myopic group, after adjustment for confounding factors $(\mathrm{p}=0.629)$.

In contrast to previous Asian studies, lower serum zinc is not associated with the presence of myopia in Korean adolescents. However, further well-designed prospective studies should be performed in a large cohort of various ethnic groups.

1. Holden BA, Fricke TR, Wilson DA et al. (2016) Global Prevalence of Myopia and High Myopia and Temporal Trends from 2000 through 2050. Ophthalmology 123(5), 1036-1042.

2. Fedor M, Socha K, Urban B et al. (2017) Serum Concentration of Zinc, Copper, Selenium, Manganese, and Cu/Zn Ratio in Children and Adolescents with Myopia. Biol Trace Elem Res 176(1), 1-9.

3. Wang L (2009) Variation analysis of six kinds of common micro- elements contents of blood in myopic primary school students in Dongguan district. Cent Chin Med J 1, 20-2. 